\title{
Review of Road Detection and Segmentation Technique Using CNN
}

\author{
${ }^{1}$ Bhawna Shokeen, ${ }^{2}$ Diksha Chawla \\ 1,2 Department of computer science And Engineering, PDM University, Bahadurgarh, Haryana, \\ India. 1bhawnashokeen91@gmail.com, 2diksha_wengg@pdm.ac.in
}

\begin{abstract}
This research proposes form shape mounted on "the deep convolutional neural network (CNN) for the detection of roads and the segmentation of aerial pix. Those images are received by using a UAV. The photograph segmentation set of rules has two levels: the studying segment and the working phase. The aerial images of the data deteriorated into their coloration additives, had been pre-processed in matlab on hue, after which divided into small 33 $\times 33$ pixel packing containers the usage of a sliding container set of rules. CNN was once designed with matconvnet and had the accompanying structure: 4 convolutional levels, 4 grouping stages, a relu layer, a totally linked layer, and a softmax layer. The entire community has been organized for the use of 2,000 boxes. CNN was implemented the use of matlab programming on the gpu and the outcomes are promising. The CNN output offers pixel-by means of-pixel records, which class it has a location with (road / non-road). White pixel and choppy terrain are known as "0" (dark). Monitoring roads is a troublesome venture in aerial picture segmentation due to quite more than a few sizes and surfaces. One of the vastest steps in CNN training is the pre-processing phase. Due to toll road segmentation, dismissal structures and complexity enhancement have been applied." this is an audited article on the relationship between representative upkeep techniques with work pleasure and responsibility in insurance plan businesses.
\end{abstract}

Keywords:Aerial image, CNN architecture, CNNS Segmentation, Image Segmentation, Road Detection, Techniques used in CNN.

\section{INTRODUCTION}

Although automata were initially developed for military purposes, in recent years they have surprisingly become the mainstream for regular use by citizens and businesses. It is relatively simple to add a camera to an automaton, considers many applications. For example, they are used as an emotional support network in firefighting exercises, as an automaton can provide a guide to move the fire forward without endangering human life. The first developed road detector focused on very much cleared Roads with precise edges.

In this example, it turned into noticeably clean to discover avenue limitations or even road signs, the use of techniques which include hough's transformation or coloration-based segmentation. However, those strategies permit for small versions within the type of street that can be detected [1]. Although transformation may have terrible performance on dust roads and rural areas, where shrubs and sand are more likely to cowl the boundary strains and will therefore complicate their detection. In flip, the main problem for colour-primarily based techniques can be the remarkable diversity of colors that a road can present relying at the lighting fixtures, fabric and conditions of the road. In addition, different objects with a avenue-like color are frequently located within the scene, ensuing in fake positives. More research has currently been executed in this location, particularly to use it in driver less motors. Although right outcomes have been completed with techniques including Segmentation vector machines (SVM) or item-primarily based model detectors, a superb improvement has been achieved with the popularization of convolutional neural networks (CNN) after the arrival of alexnet thanks to their brilliant performance; CNNs are now one of the preferred answers for image popularity. One of the recently developed $\mathrm{CNN}$-based structures is segnet, used in this report[2]."

Most by far of studies center on "the driver's point of view. However, this methodology is altogether different from what we are looking for right now; a drone catches images with an aerial view of the road. Even though there are a few ways to deal with the road following from an aerial perspective, they depend on exemplary techniques, for example, the snake histogram-based edge and line detection utilizing Hough's change or alignment of the homography and in the detection of graphic cuts."

\section{A. CNNS Segmentation}

Convolutional neural networks are presently "the high-quality deep mastering architecture for a semantic 
photograph that receives tasks. Its fundamental property is the use of layers that carry out found out convolution channels: this project considers the invariability of the movement of photo structures and reduces in in large part the quantity of loose parameters inside the version. CNNs can be received by using beginning administratively to stop the back off algorithm, which generally uses stochastic gradients in small agencies through competition. Inside the past years, they had been very fruitful and caused a small revolution in the regions of speech and photo analysis [3].

\section{B. Fully convolutional neural networks}

CNN in "its original structure turned into designed for the popularity, for instance, of assigning a unmarried label to an entire photograph. The bottleneck, whilst used for semantic segmentation (labeling of every pixel) is the loss of spatial region [4]: From one angle, convolution became redesigned, and clustering extended spatial facts and reduced its decision, consequently many regularly severe and completely related layers fuse facts from throughout the picture to generate its output.

\section{Multiple CNN Training}

Deep nets are "notorious for having incredibly large non-convex loss functions with numerous near minima. In the remote possibility of initializing with multiple parameter sets, the network has ensured to fulfill various agreements, even if they see similar training information. This insight recommends a simple media strategy model (learning collection): form some networks with multiple initialization and average their assumptions. The outcomes proves that, as seen for the image, level order, for example, the average of several CNN examples further improves performance.

\section{Pre-trained Networks}

The "CNN image analysis models have been trained in much iteration, The use of large databases with lots or even thousands and thousands of photographs. Happily, it turned out that $\mathrm{CNN}$ are useful for getting to know transfers: once a community has been skilled with a huge database, it has adapted properly enough to the photograph statistics shape in widespread, in order that it could adapt to a new venture with exceptionally negative training."

The vgg-16 network was designed to mission imageNet 2012 large-scale visual recognition and excellently designed with outstanding effects. The highlights of the $\mathrm{vgg}$ architecture are generally almost all the parameters that may be skilled according to layer, because of the usage of small 3 $x$ three convolution centers. This lets in you to put together severe networks with 16 (or maybe 19) levels in a length of affordable time. For our semantic segmentation flow, we converted the 16 layer variant into an FCN. This was the most powerful individual network for our information.

Implementation info although $\mathrm{CNN}$ offers stop-to-end device learning and achieves first rate experimental consequences; schooling calls for a few issues. In our community the part that seems to be generally difficult to learn are the layers of DE convolution. We start to upload sampling loads however; these layers moderate backward proliferation and require many training accents.

We can consider that "the network has generally finished its training multiple times. The learning rate fluctuated somewhere in the range of 0.0005 and 0.005 , depending on the time number. Figure 1 Continually speaks to within the diagram at the left the training error (blue) and the approval mistakes (green). The grain size and the quantity of channels in keeping with layer (inner and out) were extra tests and triggered better exactness of the road segmentation. This $\mathrm{CNN}$ is usually powerful to adjustments, yet works first-class in territories without bushes (masking the street) and in systems (concrete buildings or roofs).

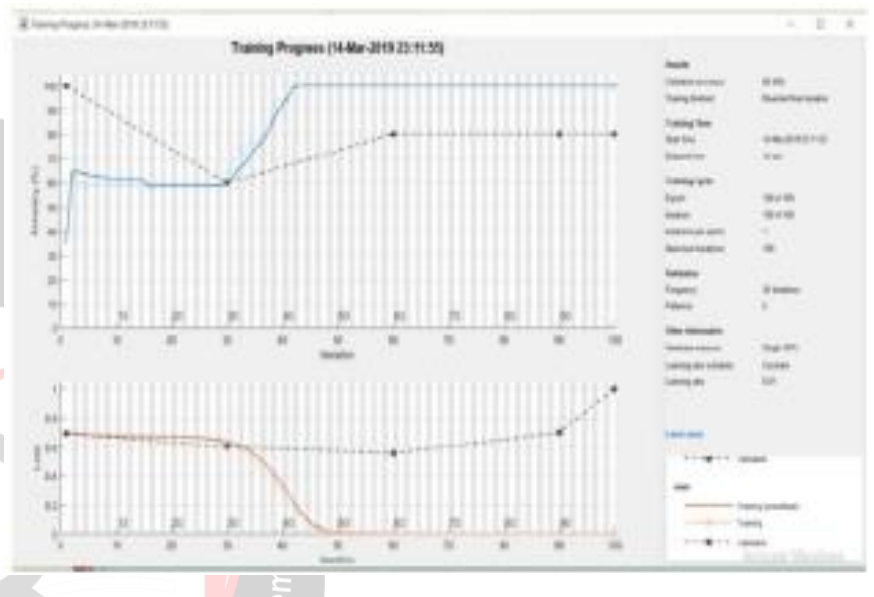

Fig. 1. Learning Rate Graph

In this research, "we will propose a system capable of Segmenting roads from aerial pictures concerned with a set-wing UAV. The device is predicated on a CNN architecture that makes use of a controlled studying set of rules. CNN was designed the usage of matconvnet. In contrast to the algorithms brought within the sleek, ours has the advantage of ease and precision.

\section{MAJOR CONTRIBUTIONS}

\section{A. System Architecture}

Detecting and "segmenting roads from aerial photography is presented in fig. 2 and consists of two predominant modules: UAV module (constant wing kind) and ground module. The pictures taken from the UAV video digital camera are transmitted through the digital data connection to the floor module. To detect and section the roads, subsequent images are received with a steady frequency within the trajectory of this system. The images are saved in the image buffer for later processing." After "the main image has been processed, the image slide window decomposes. The size of the box is $33 \times 33$ pixels with a 1 pixel cursor. In this way, the records for the proposed conference neural network are monochrome pictures (similar to the $\mathrm{h}$ issue) of $33 \times 33$ 
pixels. These are handed through the complete system to institution them into two classes: street and no-road.

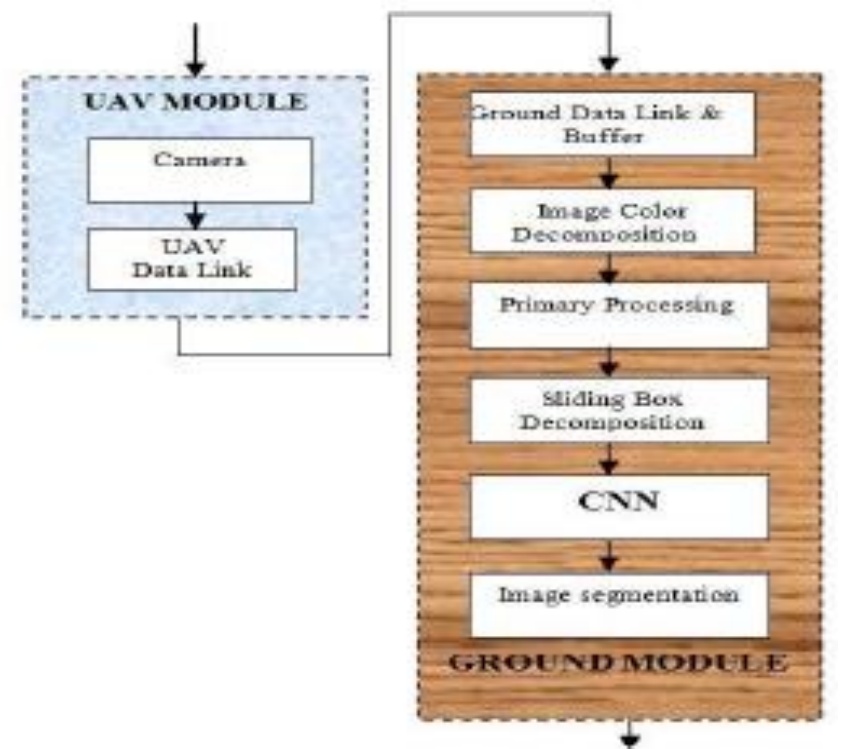

Fig. 2. System Architecture

\section{B. CNN architecture}

CNN architecture consists of "4 convolutional stages observed by way of 4 spatial reduction stages (grouping levels), a completely linked level, a relu hobby level, and a softmax stage. It is a customized neural network structure that has been directed. The info images were chosen by the UAS Carried out by using the authors inside the muros studies assignment. The result of a particular CNN operation (convolution or grouping) is characterized as an indicator map[5]. Every one of the effects received can have a third size, the profundity (the wide variety of neurons) of each layer. Community usefulness relies upon on two stages: the mastering phase and the operational phase. All the precept coping with steps implemented inside the education set will likewise be accomplished inside the check set."

In "the class manner (operational phase), CNN receives a data outline, with same dimensions from the schooling define $(33 \times 33$ pixels $)$ and returns, for the focal pixel, a rating for each class. For every pixel, we consider the elegance with the maximum noteworthy score. The pixels arranged with the aid of CNN are viewed as keep going for the recreation of fragmented images. Right now, pixels are incorporated into another fragmented image). Presently, morphological operations (disintegration and dilatation) are performed to take out the conceivable noise because of the segmentation procedure.

\section{Image segmentation}

The initial step of "the proposed manner is to fragment all of the casings of a video into special classes, in the street case, sky, and different. To do all things considered, we use segnet, deep convolutional encoder-decoder architecture with a closing pixel-clever portrayal layer, able to dividing an picture into numerous classes continuously. This framework achieves pinnacle tier performance and is educated to peer avenue scenes, which makes it a now not too horrific planning stage for our motivation. Nevertheless, we need to modify it to oblige our dispositions higher, likely [6]."

Certainly one of "the parameters to exchange are the recurrence of the presence of each elegance. This is used for sophistication adjusting, which improves the results while the variety of pixels having a niche with a degree is a good deal lesser than any other (for our scenario, the instructions street and sky are some distance less overflowing than the elegance different). “

The recurrence of look is "used for weighting every magnificence inside the neural network's botch paintings, so a goof in the grouping of a pixel having a niche with a less regular class could be more and more rebuffed. As our database and training are not equivalent to the segnet originals, the equipped class frequencies aren't intelligible with the pics used. To sign up our class frequencies, we use a comparable situation as the segnet makers,"

$$
\alpha c=\text { median } f \text { req } / f \text { req }(c)
$$

freq(c) is the number of pixels in class $c$ separated by using the number of pixels that belong to an picture where well known c seems, and median freq is the median of those frequencies.

\section{LITERATURE REVIEW}

D. Popescu and 1. Ichim (2016) [7] analyzed that "the extraction of dependable statistics from aerial snap shots is a complicated hassle. However, it has several vital makes use of: tracking of screw ups (tremors, floods, flora fires, and many others.), tracking of crops in precision horticulture, notion of margins, monitoring of visitors, etc. In aerial tracking of land surfaces, the detection and segmentation of roads face a vital take a look at. To this quit, a one of a kind photo dealing with strategies has been taken into consideration. Surface analysis techniques are used to locate and phase intrigue areas, and specifically roads, from aerial imagery; however the choice of specialist highlights depends at the precise configuration of the utility that uses it."

h. Kong, j. Y. Audibert and j. Ponce (2010) [8] "the authors also taken into consideration a regulated learning method to detect road surfaces the use of a neural community. Connected photos crafted from the photo mosaic era may be beneficial in identifying and segmenting roads. At the moment, empty spaces or location duplication's, as can seem in the mixture of photographs taken, are kept in a essential separation. UAV is a more convenient and continuously versatile recreation plan (because it always ensures unrivaled imaging objectives in adverse ecosystem situations). The non-stop image that is ready in the recordings taken by using the low / medium top 
uav (multi-helicopter type) is proposed for detection and monitoring of the road."

D. Popescu and I. Ichim (2015) [9] "the authors used the philosophy of designing the Gaussian mixture version, the shape tensor and graph cut as philosophy. Diverse Avenue highlights and data, along with stroke width, pitch, and width transformation, are consolidated to include capacity competitors on the street. Then a Gaussian mixture version is advanced to explain promising hypotheses together with the road and the manufacturing unit. To manufacture the precision and generosity of road topography in a deep convolutional neural community (CNN), it turned into used in a realistic way. For proper schooling, right now, the authors have proposed getting ready a comparable image on gpu."

Chiu, K.- Y and Lin, S F (2005) [10] Have examined that "it is straightforward to associate a video camera with a drone, which reflects on a ton of packages. For instance, they're used as a beneficial shape in firefighting interventions, as a drone can provide steering to enhance hearth without risking human lifestyles. Drones are used within the equal way for traffic analysis and to find a hard-to-locate rhythm; they are able to supply more compactness than various advances. In any case, the character of these images causes inconvenience, in view that they gift an outdoor place with a excellent status quo. The camera movements, so the manufacturing facility isn't always static; both, trade from facet to diagram. Further, the point of view of a particular article can contrast substantially, depending at the condition of the drone corresponding to the terrain."

Badrinarayanan et al., (2015) [11] Explored the "principal goal of this paintings is to come across a path from the perspective of a drone, that is essential, or notably sturdy, in a ton of applications. Photo segmentation is finished using segnet, a totally convolutional deep neural network architecture for pixel-primarily based semantic segmentation to start with educated to segment an picture into 11 training. Anyhow, the images used for the main segnet schooling are taken from the perspective of the driver of a vehicle. This calls for a sad segnet appearance at the same time as the usage of aerial imagery, so we balanced and redesigned the framework with every other database, and reduced instructions from eleven to three (avenue, sky, and extra)."

Evangelidis, G. D. Psarakis, E. Z. (2008) [12] Making a These powers to create a really particular detector to access remarkable results in one of a kind regions, the first avenue detectors that have become targeted on specially clear roads with clean edges. These days, it has been tolerably easy to find street limits or maybe road signs and symptoms, the usage of strategies like hough shift "PC see a path in an image is an amazing test. There is a large collection of natural components where you can find your way. ."
Felzenszwalb, P., Girshick, R., McAllester et al. (2010) [13] "more research has been finished to apply them to autonomous automobiles. But awesome consequences have been completed with techniques like support vector machines (svm) or model-primarily based article detectors; an incredible development has been accomplished with the advancement of convolutional neural networks (CNN) after the proximity of alexnet. Thanks to its awesome performances, cnn is currently one of the compatible answers for picture affirmation. One of the early CNN-based past due frameworks is segnet, which is in use proper now."

Krizhevsky, A., Sutskever, I. and Hinton, G. (2012) [14] "characterized a non-exclusive road, and the vehicle detector Is complex, tremendously because of the tremendous form of statistics images. If it were characterized that the photographs were received in a dependable manner from a comparative perspective or that the road situations and the state have been near, the adjustment of particular parameters within the detector would have updated the results. Anyhow, the manner of this record has been to expand an unusually traditional detector that would work in a distinct area."

Lin, Y. Saripalli, S. (2012) [15] Extended "the maximum fantastic manner of coping with toll road detection, which is operating from motive force-aspect imaging, to aerial imaging. With our exploratory effects, we display that it is feasible to discover a direction from exclusive aerial perspectives with deep learning systems, obtaining an accuracy of greater than $90 \%$. Additionally, we offer a tagged database of aerial video game plans where streets are the principle topic of intrigue, just like the automated charging device.

\section{CONCLUSION}

On this investigation we are able to gift an stop-to-stop semantic segmentation technique, "which offers cutting-edge day semantic segmentation performance in aerial pictures of the isprs semantic tagging data-set. Cnns in popular in the mean time are a mature technology that non-experts can use immediately. In language processing and laptop assessment, they've got already grow to be the usual approach for a diffusion of forecasting sports, similar to the svm boom of approximately 15 years ago."

Despite the fact that we limit our studies to the semantic segmentation of vhr aerial photos of town areas, the CNN framework and its editions are very fashionable and potentially useful for masses different statistics evaluation issues in far off sensing. In this context, it's far especially useful that the engineering of the features is not necessary for a particular spatial and spectral decision of the photograph, consequently handiest the schooling data are had to switch the complete class pipeline into a brand new activity."

The "street detection will play a crucial function in those programs, as drones are especially utilized in outside places, 
wherein roads are nearly usually gift. Therefore, in the extensive majority of recent trends involving drones, roads will likely be studied or used as a reference for vicinity. The definition of an everyday avenue and automobile detector is complex, specifically due to the extensive kind of input photographs. If it were defined that the images might constantly be captured from the same point of view or that the surrounding street and conditions have been always comparable, adjusting a few parameters within the detector might enhance the outcomes.

However, "the mission of this paintings has been to develop a very conventional detector that works in very distinct environments. On this paintings, we make bigger the maximum popular technique to road detection, that's running on photographs from the motive force's point of view, to aerial images. With our experimental results, we demonstrate that it's miles possible to locate a route from very specific aerial factors of view with deep mastering techniques, acquiring an accuracy of over 90\%. Similarly, we provide a database categorized aerial video sequences where the roads are the principle object of hobby, as well as the semi-automated device that we've developed to label it.

\section{REFERENCES}

[1] Aharon Bar Hillel, Ronen Lerner, Dan Levi, and Guy Raz. Recent progress in road and lane detection: a survey. Machine vision and applications, 25(3):727-745, 2014

[2] Yecheng Lyu, Lin Bai, and Xinming Huang. Real-time road segmentation using lidar data processing on an fpga. In Circuits and Systems (ISCAS), 2018 IEEE International Symposium on, pages 1-5. IEEE, 2018

[3] Alexandre Alahi, Kratarth Goel, Vignesh Ramanathan, Alexandre Robicquet, Li Fei-Fei, and Silvio Savarese. Social lstm: Human trajectory prediction in crowded spaces. In Proceedings of the IEEE Conference on Computer Vision and Pattern Recognition, pages 961-971, 2016.

[4] Sik-Ho Tsang, (2018), "Review: FCN — Fully Convolutional Network (Semantic Segmentation)", available at:

https://towardsdatascience.com/review-fcn-semantic-segmen tation-eb8c9b50d2d1

[5] M. Sokolova and G. Lapalme, "A systematic analysis of performance measures for classification Inf. Process. Manage., vol. 45, pp.427-437, July 2009

[6] P. Kaiser, J.D. Wegner, A. Lucchi, M. Jaggi, T. Hofmann, and K. Schindler, "Learning aerial image segmentation from online maps," IEEE Transactions on Geoscience and Remote Sensing, vol. 55(11), pp.60546068, 2017

[7] D.Popescu and L. Ichim (2016), "Aerial image segmentation by use of textural features," In Proc. 20th International Conference on System Theory, Control and Computing (ICSTCC), Sinaia, Romania, pp. 721-726
[8] H. Kong, J.Y. Audibert, and J. Ponce, "General road detection from a single image," in IEEE Trans. Image Process. vol. 19, pp. 2211-2220, 2010

[9] D. Popescu and L. Ichim, "Image recognition in UAV application based on texture analysis," in ACIVS 2015. LNCS, Springer, Heidelberg, vol. 9386, pp. 693-704, 2015

[10] Chiu, K.-Y. and Lin, S.-F. (2005). Lane detection using color-based segmentation. pages 706-711.

[11] Badrinarayanan, V., Kendall, A., and Cipolla, R. (2015). SegNet: A Deep Convolutional Encoder-Decoder Architecture for Image Segmentation. CoRR, abs/1511.0.

[12] Evangelidis, G. D. and Psarakis, E. Z. (2008). Parametric image alignment using enhanced correlation coefficient maximization. IEEE Transactions on Pattern Analysis and Machine Intelligence, 30(10):1858- 1865

[13] Felzenszwalb, P., Girshick, R., McAllester, D., and Ramanan, D. (2010). Object detection with discriminatively trained part based models. IEEE Trans. Pattern Analysis and Machine Intelligence, 32(9):1627-1645

[14] Krizhevsky, A., Sutskever, I., and Hinton, G. (2012). Imagenet classification with deep convolutional neural networks. Proceedings of Neural Information and Processing Systems.

[15] Lin, Y. and Saripalli, S. (2012). Road detection from aerial imagery. In 2012 IEEE International Conference on Robotics and Automation, pages 3588-3593.Aharon Bar Hillel, Ronen Lerner, Dan Levi, and Guy Raz. Recent progress in road and lane detection: a survey. Machine vision and applications, 25(3):727-745, 2014 